\title{
Outliers Robustness in Multivariate Orthogonal Regression
}

\author{
Giuseppe Carlo Calafiore
}

\begin{abstract}
This paper deals with the problem of multivariate affine regression in the presence of outliers in the data. The method discussed is based on weighted orthogonal least squares. The weights associated with the data satisfy a suitable optimality criterion and are computed by a two-step algorithm requiring a RANSAC step and a gradient-based optimization step. Issues related to the breakdown point of the method are discussed, and examples of application on various real multidimensional data sets are reported in the paper.
\end{abstract}

Index Terms-Computer vision, influential observations, orthogonal least squares, outliers, pattern recognition, regression analysis.

\section{INTRODUCTION}

$\mathbf{T}$ HE purpose of regression analysis is to fit equations to observed data. Affine regression, in particular, deals with the problem of determining an affine relation among a set of noisy multidimensional data points (see [2]). This problem has of course a very long history, dating back to Gauss and the Least Squares (LS) method, and is still playing a key role in a number of engineering endeavors such as numerical modeling, quality control, computer vision, and statistical data analysis in general.

More recently, issues related to the sensitivity of a regression method with respect to inconsistent data (often referred to as outliers or gross error data) led to the introduction of the so called robust regression methods. Robust regression is concerned with developing estimators that are not strongly affected by outliers and are insensitive to the underlying noise distribution. The theory of multidimensional robust estimators was developed in the seventies and the basic robust estimators are classified as M-estimators, R-estimators, and L-estimators [7]. The widely used M-estimators, in particular, minimize the sum of a symmetric positive definite unimodal function $\rho\left(r_{i}\right)$ of the estimation residuals $r_{i}$ [5].

This class of robust estimators, however, do not perform well with respect to the concept of breakdown point. The breakdown point of a regression method is defined as the smallest relative amount of outlier contamination that may force the value of the estimate outside an arbitrary range. For the simple LS estimator, the breakdown point is zero, meaning that one single outlier is sufficient to move the estimate outside any predefined bound. For the class of M-estimators, the breakdown point

\footnotetext{
Manuscript received April 16, 1999; revised June 1, 2000. This paper was recommended by Associate Editor M.S. Obaidat.

The author is with the Dipartimento di Automatica e Informatica, Politecnico di Torino, 10129 Torino, Italy.

Publisher Item Identifier S 1083-4427(00)07048-X.
}

is still zero [13], while generalized M-estimators (GM-estimators) have breakdown point no better than a value that decreases with the number of regression coefficients [11]. A robust estimator achieving breakdown point 0.5 (which is the highest possible value for breakdown) is the Least Median of Squares (LMedS) estimator [13], which minimizes the median of the squared residuals. Although LMedS is now widely used in applications, some problems and limitations of the method have been pointed out in the literature. In particular, the robustness of LMedS is contingent upon the existence of at least one subset of the data carrying the correct (i.e., error free) model; when noise corrupts all the data, as in the gaussian case, the quality of the initial model estimate degrades and could lead to incorrect decisions. This problem is also common to regression methods based on the RANSAC paradigm (see the survey [12] for a comparison between LMedS and RANSAC) which is discussed in some detail in the sequel. Also, the convergence rate of LMedS is critical for large data sets, as the algorithm examines all the possible combinations of pairs of observations and computes an estimator for each combination, which includes performing a computer sort.

Much less research attention has been devoted to robust orthogonal regression, which is the subject of this paper. In this area, the recent studies of [14] and [15] focus the attention on case deletion diagnostic and influence measures for the deletion of one observation at a time. In this paper, we examine the problem of multidimensional orthogonal regression and address the issue of robustness introducing the Optimal Weighted Orthogonal Least Squares (OWOLS) algorithm, which provides high breakdown point maintaining the gaussian efficiency of LS regression methods and yielding the optimal weights as a by-product of the regression procedure. The so-found optimal weights represent influence coefficients of the data points on the final estimation residual, and take into account simultaneous deletion of multiple observations. The proposed technique is then tested on various multidimensional real data sets found in the literature.

The paper is organized as follows. Section II contains preliminary results and a closed-form solution to the $k$-dimensional weighted orthogonal fitting problem. In Section III, the main result concerning the optimal robust estimator is presented, while examples of application of the proposed methodology to several real data sets are presented in Section IV.

\section{WEIGHTED ORTHOGONAL LS REGRESSION}

In this section, we present a closed-form solution to the problem of determining the $k$-dimensional affine set closest in the Orthogonal Least Squares (OLS) sense to a set of $N$ 
TABLE I

WeISS Two DimENSIONAL

DATA

\begin{tabular}{rrr|crr|crr}
\hline$i$ & $x_{1}$ & $x_{2}$ & $i$ & $x_{1}$ & $x_{2}$ & $i$ & $x_{1}$ & $x_{2}$ \\
\hline 1 & -0.9 & 1.3 & 10 & -0.3 & 1.5 & 19 & 0.6 & 0.6 \\
2 & -0.9 & 1.4 & 11 & -0.2 & 1.4 & 20 & 0.9 & 0.7 \\
3 & -0.8 & 1.4 & 12 & -0.1 & 1.3 & 21 & 0.9 & 0.6 \\
4 & -0.8 & 1.3 & 13 & 0 & 1.2 & 22 & 0.7 & 0.5 \\
5 & -0.9 & 1.7 & 14 & 0.1 & 1.1 & 23 & 0.8 & 0.4 \\
6 & -0.9 & 1.8 & 15 & 0.2 & 1 & 24 & 0.9 & 0.3 \\
7 & -0.6 & 1.8 & 16 & 0.3 & 0.9 & 25 & 0.5 & 1.8 \\
8 & -0.5 & 1.7 & 17 & 0.4 & 0.8 & 26 & 0.6 & 1.2 \\
9 & -0.4 & 1.6 & 18 & 0.6 & 0.7 & $*$ & $*$ & $*$ \\
\hline
\end{tabular}

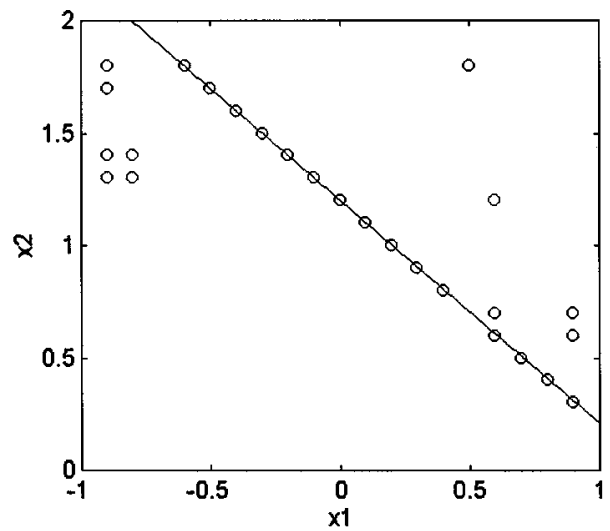

Fig. 1. Plot of Weiss data and OWOLS regression line.

points in $\mathbb{R}^{n}$. This result is related to the Total Least Squares (TLS) problem [4], [17] and it is also known in Computer Vision ([16] gives an alternative proof for the particular case of subspaces-affine sets through the origin-and unitary weights).

Let $X \in \mathbb{R}^{n \times N}$ be a matrix containing by columns the $N$ observed data $x_{i} \in \mathbb{R}^{n}, i=1, \cdots, N$ and let a $k$-dimensional affine set be described by

$$
\mathcal{F}_{k}=\left\{x \in \mathbb{R}^{n}: A^{T} x=b ; A \in \mathcal{Q}^{n \times m}, b \in \mathbb{R}^{m}\right\}
$$

where $m=n-k$, and $\mathcal{Q}^{n \times m}$ is the set of matrices in $\mathbb{R}^{n \times m}$ whose columns form an orthonormal set. From the definition, it follows for instance that in a $n$-dimensional space $\mathcal{F}_{n-1}$ represents a hyperplane, $\mathcal{F}_{1}$ represents a line and $\mathcal{F}_{0}$ represents a point.

If nonnegative weights $w_{i} \geq 0$ are associated to each data point, the weighted OLS (WOLS) problem is posed as

$$
\left(A^{*}, b^{*}\right)=\arg \min _{A \in \mathcal{Q}^{n \times m}, b \in \mathbf{R}^{m}} f(A, b, X, w)
$$

where

$$
\begin{aligned}
f(A, b, X, w) & =\sum_{i=1}^{N} w_{i}^{2}\left\|A^{T} x_{i}-b\right\|^{2} \\
& =\left\|\left(A^{T} X-b u^{T}\right) W\right\|_{F}^{2}
\end{aligned}
$$

with $w=\left[w_{1}, w_{2}, \cdots, w_{N}\right]^{T}, u \in \mathbb{R}^{N}=[1,1, \cdots, 1]^{T}, W=$ $\operatorname{diag}\{w\}$, and the subscript $F$ indicates the Frobenius matrix
TABLE II

OPTIMAL WEIGHTS FOR THE WEISS DATA

\begin{tabular}{rr|cr|cr}
\hline$i$ & $\bar{w}_{i}$ & $i$ & $\bar{w}_{i}$ & $i$ & $\bar{w}_{i}$ \\
\hline 1 & 0.0097 & 10 & 1.0000 & 19 & 1.0000 \\
2 & 0.0127 & 11 & 1.0000 & 20 & 0.0403 \\
3 & 0.0173 & 12 & 1.0000 & 21 & 0.0721 \\
4 & 0.0127 & 13 & 1.0000 & 22 & 1.0000 \\
5 & 0.0392 & 14 & 1.0000 & 23 & 1.0000 \\
6 & 0.0706 & 15 & 1.0000 & 24 & 1.0000 \\
7 & 1.0000 & 16 & 1.0000 & 25 & 0.0051 \\
8 & 1.0000 & 17 & 1.0000 & 26 & 0.0175 \\
9 & 1.0000 & 18 & 0.7025 & $*$ & $*$ \\
\hline
\end{tabular}

\begin{tabular}{|c|c|c|c|c|c|c|c|c|c|c|c|}
\hline$i$ & $x_{1}$ & $x_{2}$ & $x_{3}$ & $x_{i}$ & $\bar{w}_{i}$ & $i$ & $x_{1}$ & $x_{2}$ & $x_{3}$ & $x_{4}$ & $\bar{w}_{i}$ \\
\hline 1 & 10.1 & 19.6 & 28.3 & 9.7 & 0.0061 & 39 & 2.1 & 0 & 1.2 & -0.7 & 1.0000 \\
\hline 2 & 9.5 & 20.5 & 28.9 & 10.1 & 0.0056 & 40 & 0.5 & 2 & 1.2 & -0.5 & 1.0000 \\
\hline 3 & 10.7 & 20.2 & 31 & 10.3 & 0.0055 & 41 & 3.4 & 1.6 & 2.9 & -0.1 & 1.0000 \\
\hline 4 & 9.9 & 21.5 & 31.7 & 9.5 & 0.0061 & 42 & 0.3 & 1 & 2.7 & -0.7 & 1.0000 \\
\hline 5 & 10.3 & 21.1 & 31.1 & 10 & 0.0057 & 43 & 0.1 & 3.3 & 0.9 & 0.6 & 0.7641 \\
\hline 6 & 10.8 & 20.4 & 29.2 & 10 & 0.0059 & 44 & 1.8 & 0.5 & 3.2 & -0.7 & 1.0000 \\
\hline 7 & 10.5 & 20.9 & 29.1 & 10.8 & 0.0050 & 45 & 1.9 & 0.1 & 0.6 & -0.5 & 1.0000 \\
\hline 8 & 9.9 & 19.6 & 28.8 & 10.3 & 0.0054 & 46 & 1.8 & 0.5 & 3 & -0.4 & 1.0000 \\
\hline 9 & 9.7 & 20.7 & 31 & 9.6 & 0.0060 & 47 & 3 & 0.1 & 0.8 & -0.9 & 0.4401 \\
\hline 10 & 9.3 & 19.7 & 30.3 & 9.9 & 0.0054 & 48 & 3.1 & 1.6 & 3 & 0.1 & .0000 \\
\hline 11 & 11 & 24 & 35 & -0.2 & 1.0000 & 49 & 3.1 & 2.5 & 1.9 & 0.9 & 1.0000 \\
\hline 12 & 12 & 23 & 37 & -0.4 & 1.0000 & 50 & 2.1 & 2.8 & 2.9 & -0.4 & 1.0000 \\
\hline 13 & 12 & 26 & 34 & 0.7 & 1.0000 & 51 & 2.3 & 1.5 & 0.4 & 0.7 & 1.0000 \\
\hline 14 & 11 & 34 & 34 & 0.1 & 1.0000 & 52 & 3.3 & 0.6 & 1.2 & -0.5 & 0.8960 \\
\hline 15 & 3.4 & 2.9 & 2.1 & -0.4 & 0.9753 & 53 & 0.3 & 0.4 & 3.3 & 0.7 & 0.3011 \\
\hline 16 & 3.1 & 2.2 & 0.3 & 0.6 & 1.0000 & 54 & 1.1 & 3 & 0.3 & 0.7 & 1.0000 \\
\hline 17 & 0 & 1.6 & 0.2 & -0.2 & 1.0000 & 55 & 0.5 & 2.4 & 0.9 & 0 & 1.0000 \\
\hline 18 & 2.3 & 1.6 & 2 & 0 & 1.0000 & 56 & 1.8 & 3.2 & 0.9 & 0.1 & 1.0000 \\
\hline 19 & 0.8 & 2.9 & 1.6 & 0.1 & 1.0000 & 57 & 1.8 & 0.7 & 0.7 & 0.7 & 1.0000 \\
\hline 20 & 3.1 & 3.4 & 2.2 & 0.4 & 1.0000 & 58 & 2.4 & 3.4 & 1.5 & -0.1 & 1.0000 \\
\hline 21 & 2.6 & 2.2 & 1.9 & 0.9 & 1.0000 & 59 & 1.6 & 2.1 & 3 & -0.3 & 1.0000 \\
\hline 22 & 0.4 & 3.2 & 1.9 & 0.3 & 1.0000 & 60 & 0.3 & 1.5 & 3.3 & -0.9 & 1.0000 \\
\hline 23 & 2 & 2.3 & 0.8 & -0.8 & 0.6553 & 61 & 0.4 & 3.4 & 3 & -0.3 & 1.0000 \\
\hline 24 & 1.3 & 2.3 & 0.5 & 0.7 & 1.0000 & 62 & 0.9 & 0.1 & 0.3 & 0.6 & 0.8883 \\
\hline 25 & 1 & 0 & 0.4 & -0.3 & 1.0000 & 63 & 1.1 & 2.7 & 0.2 & -0.3 & 1.0000 \\
\hline 26 & 0.9 & 3.3 & 2.5 & -0.8 & 1.0000 & 64 & 2.8 & 3 & 2.9 & -0.5 & 1.0000 \\
\hline 27 & 3.3 & 2.5 & 2.9 & -0.7 & 0.6761 & 65 & 2 & 0.7 & 2.7 & 0.6 & 0.9491 \\
\hline 28 & 1.8 & 0.8 & 2 & 0.3 & 1.0000 & 66 & 0.2 & 1.8 & 0.8 & -0.9 & 1.0000 \\
\hline 29 & 1.2 & 0.9 & 0.8 & 0.3 & 1.0000 & 67 & 1.6 & 2 & 1.2 & -0.7 & 1.0000 \\
\hline 30 & 1.2 & 0.7 & 3.4 & -0.3 & 1.0000 & 68 & 0.1 & 0 & 1.1 & 0.6 & 0.4791 \\
\hline 31 & 3.1 & 1.4 & 1 & 0 & 1.0000 & 69 & 2 & 0.6 & 0.3 & 0.2 & 1.0000 \\
\hline 32 & 0.5 & 2.4 & 0.3 & -0.4 & 1.0000 & 70 & 1 & 2.2 & 2.9 & 0.7 & 0.5188 \\
\hline 33 & 1.5 & 3.1 & 1.5 & -0.6 & 1.0000 & 71 & 2.2 & 2.5 & 2.3 & 0.2 & 1.0000 \\
\hline 34 & 0.4 & 0 & 0.7 & -0.7 & 1.0000 & 72 & 0.6 & 2 & 1.5 & -0.2 & 1.0000 \\
\hline 35 & 3.1 & 2.4 & 3 & 0.3 & 1.0000 & 73 & 0.3 & 1.7 & 2.2 & 0.4 & 0.7570 \\
\hline 36 & 1.1 & 2.2 & 2.7 & -1 & 1.0000 & 74 & 0 & 2.2 & 1.6 & -0.9 & 1.0000 \\
\hline 37 & 0.1 & 3 & 2.6 & -0.6 & 1.0000 & 75 & 0.3 & 0.4 & 2.6 & 0.2 & 0.8942 \\
\hline 38 & 1.5 & 1.2 & 0.2 & 0.9 & 0.7477 & & & & & & \\
\hline
\end{tabular}

TABLE III

HaWkins, BRADU AND KaSs Four DimENSIONAL Data, AND OPTIMAL WEIGHTS $\bar{w}$. FROM OWOLS REGRESSION

norm $\left(\|Y\|_{F}^{2}=\operatorname{Tr}\left(Y Y^{\prime}\right)=\operatorname{Tr}\left(Y^{\prime} Y\right)\right.$, where $\operatorname{Tr}(\cdot)$ is the trace operator). The following proposition characterizes the solution of the WOLS regression problem.

Proposition 1: The optimal solution $\left(A^{*}, b^{*}\right)$ of (2) is given by

$$
A^{*}=\left[a_{1}^{*}, a_{2}^{*}, \cdots, a_{m}^{*}\right] ; \quad b^{*}=\frac{1}{\nu} A^{* T} X W^{2} u
$$

where $a_{1}^{*}, a_{2}^{*}, \cdots, a_{m}^{*}$ are the $m$ unit norm eigenvectors associated with the first $m$ smallest eigenvalues of the positive semidefinite matrix

$$
H=X\left(W^{2}=\frac{1}{\nu} W^{2} u u^{T} W^{2}\right) X^{T}
$$


and $\nu=\sum_{i=1}^{N} w_{i}^{2}$. The value of the objective function at the optimum is given by

$$
f^{*}(X, w)=f\left(A^{*}, b^{*}, X, w\right)=\sum_{i=1}^{m} \lambda_{i}
$$

where $\lambda_{i}$ are the eigenvalues of $H$ arranged in increasing order.

Proof: See Appendix A.

The regression estimate found in (4) will be referred to as WOLS regressor, while in the case of unitary weights, we recover the standard OLS regression. In the following section, we consider the variation of the value (6) of the objective function at an optimal point, with respect to variations of the weights vector. This will lead to an "influence-adjustment" scheme where a set of suitably computed optimal weights provide information about the influence on the regression of each observation. Data corresponding to low weights are influential observations for the regression that can potentially be removed from the sample space.

\section{OPTIMAL WeIGHTED OLS}

The WOLS estimator introduced in the previous section is of course not a robust regressor, sinceit is based on LS minimization having zero breakdown point, as previously discussed. Consider now the following estimator (OWOLS)

$$
(\bar{A}, \bar{b})=\arg \min _{A \in \mathcal{Q}^{n \times m}, b \in \mathbf{R}^{m}} f(A, b, X, \bar{w})
$$

where

$$
\begin{gathered}
\bar{w}=\arg \min _{w \in \mathbb{R}^{N}} f^{*}(X, w) \\
\text { subject to: } \\
\sum_{i=1}^{N} w_{i} \geq N-N_{o}, \\
w_{i} \in[0,1] ; \quad \forall i .
\end{gathered}
$$

In (9), $N_{\circ}$ is an estimated upper bound on the number of outliers present in the data. Clearly, OWOLS is still a weighted OLS estimator, where the weights $\bar{w}$ are computed so to satisfy the optimality condition (8) under the constraints (9), (10). A vector $w$ satisfying (9), (10) will be called in the sequel a feasible weight vector. The usual definition of breakdown point [1] is based on the variation of the regression coefficients vector returned by the regression estimator; this is no longer practical in our general case, as a matrix $A$ and a vector $b$ are returned by the OWOLS estimator. Let us introduce the following measure of robustness-denoted in the sequel as residual breakdown point - defined in terms of the final regression residual $\bar{f}(X)$, which is the value of the objective function in (7) at the optimum

$$
\bar{f}(X)=f(\bar{A}, \bar{b}, X, \bar{w}) .
$$

Let $Z \in \mathbb{R}^{n \times N}$ be any sample of $N$ observations, and let $\bar{f}(Z)$ be the value of the final regression residual when a regression estimator $\mathcal{T}$ is applied to $Z$. Consider now all possible corrupted

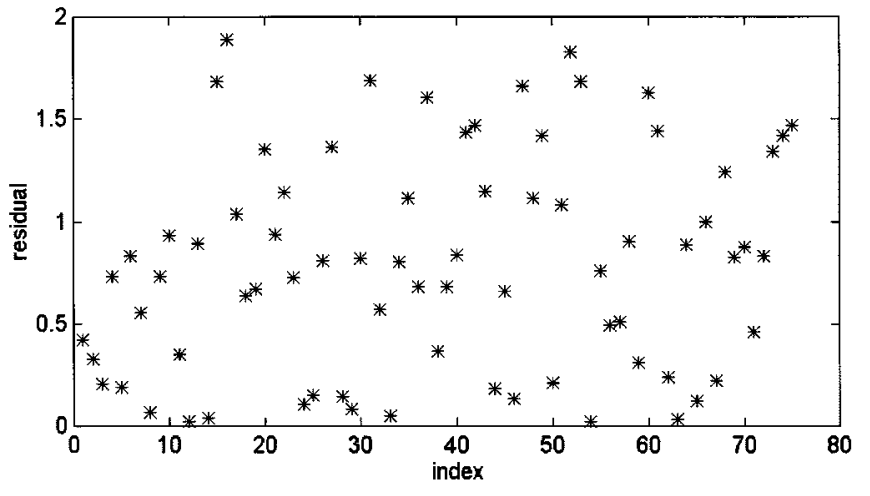

Fig. 2. Residual plot of the OLS estimator on the Hawkins, Bradu and Kass data. $A^{T}=[0.9567,0.0106,-0.2895,-0.0265], b=1.0000$. Final estimation error $f^{*}=67.63$.

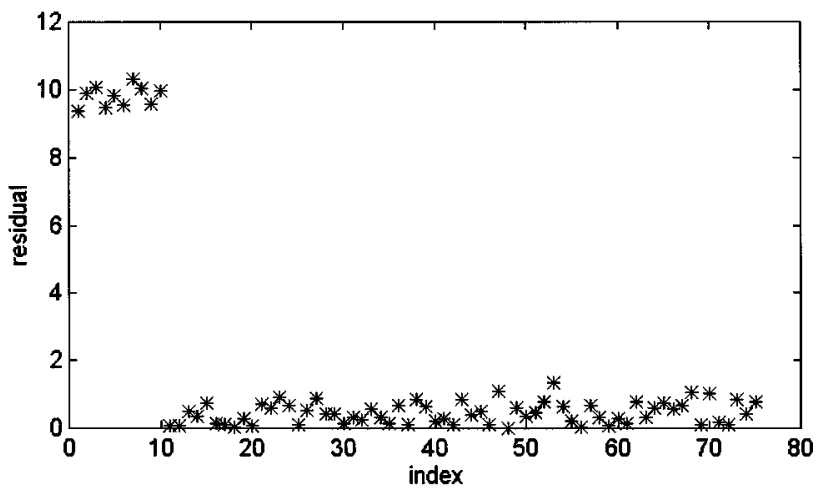

Fig. 3. Residual plot of the OWOLS estimator on the Hawkins, Bradu and Kass data. $A^{T}=[-0.2360,-0.0609,0.1128,0.9633], b=-0.3852$. Final estimation error $\bar{f}=13.08$.

samples $\tilde{Z}$ obtained upon replacing any $p$ of the original observations by arbitrary values. The residual breakpoint $\epsilon(\mathcal{T}, Z)$ is defined as

$$
\epsilon(\mathcal{T}, Z)=\min \left\{\frac{p}{N}: \sup _{\tilde{Z}}|\bar{f}(Z)-\bar{f}(\tilde{Z})|=\infty\right\} .
$$

Proposition 2: For $N_{o}<0.5 N$, the residual breakdown point of the OWOLS regression estimator is $\epsilon=N_{o} / N$.

Proof: We show that for any $p<N_{o},|\bar{f}(Z)-\bar{f}(\tilde{Z})|$ is bounded for any choice of $\tilde{Z}$. Let $\bar{w}$ be the global minimum solution of (8), then

$$
\bar{f}(Z)=f^{*}(Z, \bar{w}) \leq f^{*}(Z, w), \quad \forall \text { feasible } w .
$$

If one defines $f_{\text {sup }}=\sup f^{*}(Z, w)$ over all feasible $w$, then

$$
f^{*}(Z, w) \leq f_{\text {sup }}, \quad \forall \text { feasible } w .
$$

For any corrupted sample $\tilde{Z}$, let $\tilde{w}$ be a vector of weights having zero at the $p$ locations of the corrupted observations and one elsewhere, then $\tilde{w}$ is feasible and

$$
f^{*}(Z, \tilde{w})=f^{*}(\tilde{Z}, \tilde{w}) \geq \bar{f}(\tilde{Z}) .
$$

From (14) and (15), it follows that

$$
\begin{aligned}
|\bar{f}(Z)-\bar{f}(\tilde{Z})| \leq \mid & |\bar{f}(Z)|+|\bar{f}(\tilde{Z})| \leq|\bar{f}(Z)| \\
& +\left|f^{*}(Z, \tilde{w})\right| \leq|\bar{f}(Z)|+f_{\text {sup }}
\end{aligned}
$$


TABLE IV

AIRCRAFT DATA AND OPTIMAL WEIGHTS $\bar{w}_{i}$ FROM THE OWOLS ESTIMATOR

\begin{tabular}{r|rrrrr||r}
\hline$i$ & $x_{1}$ & $x_{2}$ & $x_{3}$ & $x_{4}$ & $x_{5}$ & $\bar{w}_{i}$ \\
\hline 1 & 6.3 & 1.7 & 8176 & 4500 & 2.76 & 1.0000 \\
2 & 6.0 & 1.9 & 6699 & 3120 & 4.76 & 1.0000 \\
3 & 5.9 & 1.5 & 9663 & 6300 & 8.75 & 1.0000 \\
4 & 3.0 & 1.2 & 12837 & 9800 & 7.78 & 1.0000 \\
5 & 5.0 & 1.8 & 10205 & 4900 & 6.18 & 1.0000 \\
6 & 6.3 & 2.0 & 14890 & 6500 & 9.50 & 0.9403 \\
7 & 5.6 & 1.6 & 13836 & 8920 & 5.14 & 0.8037 \\
8 & 3.6 & 1.2 & 11628 & 14500 & 4.76 & 1.0000 \\
9 & 2.0 & 1.4 & 15225 & 14800 & 16.70 & 1.0000 \\
10 & 2.9 & 2.3 & 18691 & 10900 & 27.68 & 1.0000 \\
11 & 2.2 & 1.9 & 19350 & 16000 & 26.64 & 1.0000 \\
12 & 3.9 & 2.6 & 20638 & 16000 & 13.71 & 1.0000 \\
13 & 4.5 & 2.0 & 12843 & 7800 & 12.31 & 1.0000 \\
14 & 4.3 & 9.7 & 13384 & 17900 & 15.73 & 0.0040 \\
15 & 4.0 & 2.9 & 13307 & 10500 & 13.59 & 0.1697 \\
16 & 3.2 & 4.3 & 29855 & 24500 & 51.90 & 1.0000 \\
17 & 4.3 & 4.3 & 29277 & 30000 & 20.78 & 1.0000 \\
18 & 2.4 & 2.6 & 24651 & 24500 & 29.82 & 1.0000 \\
19 & 2.8 & 3.7 & 28539 & 34000 & 32.78 & 1.0000 \\
20 & 3.9 & 3.3 & 8085 & 8160 & 10.12 & 0.0476 \\
21 & 2.8 & 3.9 & 30328 & 35800 & 27.84 & 1.0000 \\
22 & 1.6 & 4.1 & 46172 & 37000 & 107.10 \\
23 & 3.4 & 2.5 & 17836 & 19600 & 11.19 & 0.0346 \\
\hline
\end{tabular}

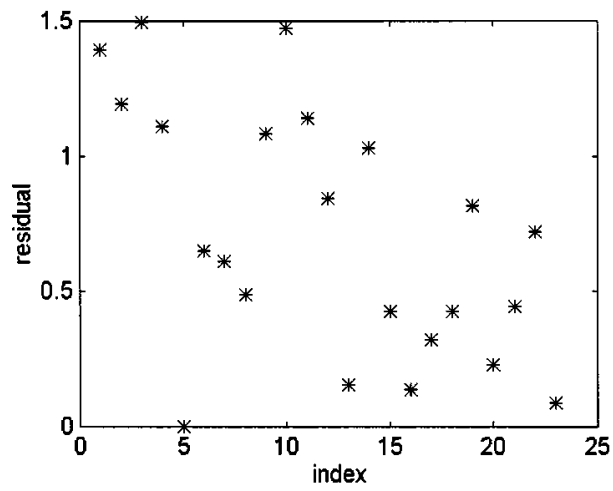

Fig. 4. Residual plots of the OLS estimator on the aircraft data.

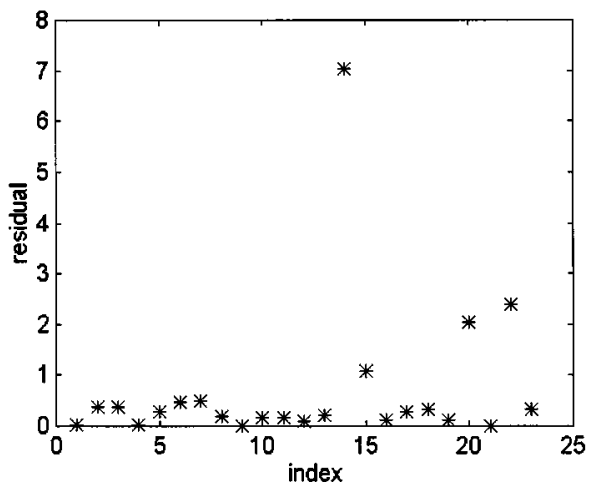

Fig. 5. Residual plots of the OWOLS estimator on the aircraft data.

therefore $|\bar{f}(Z)-\bar{f}(\tilde{Z})|$ is bounded for any corrupted sample $\tilde{Z}$.

Proposition 2 indicates that if a global solution of (8) is found, the resulting OWOLS estimate has a residual breakdown point as high as 0.5 . The requirement $N_{o}<0.5 N$ is needed because no robust regression method can work if more than half of the data is corrupted by outliers, see for instance [13]. Notice also that each weight $w_{i}$ is constrained in the interval $[0,1]$, and that the constraint (9) implies that no more than $N_{o}$ observations can be assigned a zero weight, that is no more than $N_{O}$ observations may be removed from the sample space. A method for the numerical solution of (8) is discussed in the next section.

\section{A. Computing the Optimal Weights}

Determining the optimal weight vector $w$ requires the numerical solution of the nonlinear optimization problem (8). The proposed procedure is divided into two steps.

Step 1: Use the RANSAC paradigm on the data to obtain a crude estimate of the number of influential observations $N_{o}$, and an initial feasible vector of weights $w_{0}$.

Step 2: Use a gradient based optimization algorithm to solve (8) for the optimal weights, starting for the given initial feasible point $w_{0}$.

Step 1: RANSAC (Random Sample Consensus) is a well known smoothing procedure [3] which uses as small an initial data set as feasible to instantiate a model, and enlarges this set with consistent data when possible. In the problem at hand, the minimum number of observations needed to determine a $k$-dimensional affine set is given by $n_{p}=k+1$, and the RANSAC procedure is applied as follows.

- Randomly select a subset $X^{(1)}$ of $n_{p}$ observations from $X$ and use $X^{(1)}$ to instantiate a model.

- Determine the subset $X^{(2)}$ (the consensus set) of data that are within a $\delta$-error tolerance from the instantiated model.

- Repeat the above steps $N_{s}$ times and exit with the consensus set of greatest cardinality, $X^{(*)}$.

- Determine a new model based on the data in $X^{(*)}$ and rank the observations with respect to their residuals $r_{i}$ from this model. A threshold on the residuals is used to estimate the number of outliers $N_{O}$.

- An initial feasible weight vector $w_{0}$ is determined as $w_{0, i}=\alpha e^{-r_{i} / \beta}$, where $\alpha, \beta$ are real parameters that are numerically determined such that the resulting $w_{0}$ is feasible, that is

$$
\sum_{i=1}^{N} w_{0, i} \geq N-N_{o} ; \quad w_{0, i} \in[0 ; 1] .
$$

The unspecified parameters used in the above procedure, namely the tolerance $\delta$ used to determine whether a point is compatible with a model and the number $N_{s}$ of random subsets to try, are problem dependent and clear indications on how to choose them are reported in [3].

Step 2: Once the initial feasible weight vector $w_{0}$ is given, any gradient based optimization method for non linear problems under linear constraints may be used to solve (8). In particular, closed form expressions for the gradient of the objective function $f^{*}(X, w)$ with respect to the weights are here derived as follows. Recalling (6), we have that

$$
\begin{aligned}
f^{*}(X, w) & =\sum_{i=1}^{m} \lambda_{i}(H) \\
H & =X\left(W^{2}-\frac{1}{\nu} W^{2} u u^{T} W^{2}\right) X^{T}
\end{aligned}
$$


therefore

$$
\frac{\partial f^{*}}{\partial w_{j}}=\sum_{i=1}^{m} \frac{\partial \lambda_{i}(H)}{\partial w_{j}}
$$

Using a standard formula for eigenvalues derivatives [8] we have that

$$
\frac{\partial \lambda_{i}(H)}{\partial w_{j}}=h_{i}^{T} \frac{\partial H}{\partial w_{j}} h_{i}
$$

where $h_{i}$ is the unit norm eigenvector associated with $\lambda_{i}$. The matrix derivative in (17) has the following expression

$$
\frac{\partial H}{\partial w_{j}}=2 w_{j}\left(x_{j} x_{j}^{T}+\frac{1}{\nu^{2}}\left(X w^{2}\right)\left(X w^{2}\right)^{T}-\frac{1}{\nu}\left(q_{j}+q_{j}^{T}\right)\right)
$$

where $q_{j}=X w^{2} x_{j}^{T}$, and $w^{2}=\left[w_{1}^{2}, w_{2}^{2}, \cdots, w_{N}^{2}\right]^{T}$.

\section{EXAMPLES}

In this section, we describe the application of the presented procedure to the analysis of several real data sets encountered in the literature. We start with a two-dimensional (2-D) line-fitting example presented in [9] and [18]; the data are listed in Table I and displayed in Fig. 1.

Assuming an a priori fraction of outlier contamination equal to $\gamma=30 \%$, the a priori probability that a randomly selected data point is an inlier is equal to $\eta=1-\gamma=0.7$. The estimated number $N_{s}$ of subsets trials ensuring with probability $z=0.95$ that at least one of the selected subsets is an in-model set is approximately $N_{s}=3 \eta^{-n_{p}} \simeq 6$ (see [3]). The $N_{s}$ RANSAC trials yielded a best consensus set of cardinality 16. Data from the consensus set are then used to instantiate a new model, from which the estimate $N_{o}=10$ and the initial weight vector $w_{0}$ are obtained. The numerical solution of (8) with starting point $w_{0}$ yielded the optimal weight vector $\bar{w}$ listed in Table II. The optimal regression parameters are $A^{T}=[-0.7046,-0.7096]$, $b=-0.8531$, and the regression line is shown in Fig. 1 .

In this 2-D setting, the problem may of course seem to be "overkilled," as the outlying points can easily be spotted by plotting the data. This is no longer true for data sets in spaces of dimension higher than three. It should be emphasized that in the multidimensional case, even the analysis of the scatter (or residual) plot does not help in general in discovering regression outliers that are leverage points (points with very high effect on the estimator, capable of tilting the estimate, see [13].

We next analyze the data generated by Hawkins et al.[6] for illustrating the merits of a robust technique. This artificial data set offers the advantage that at least the position of the bad points is known, which avoids some of the controversies that are inherent in the analysis of real data. The Hawkins, Bradu and Kass data set (Table III) consists of 75 observations in four dimension. The first ten observations are bad leverage points while the remaining observations are good data. The problem is in this case to fit a hyperplane (affine set of dimension $k=n-1$ ) to the observed data. In Fig. 2, we plot the regression residuals from the model obtained from the standard OLS estimator: the leverage point data are masked and do not show up from the residual plot.
Application of the RANSAC procedure (Step 1) to these data yields an estimated number of outliers $N_{o}=14$ and an initial weights vector $w_{0}$. Solving (8) (Step 2) eventually gives the optimal weight vector $\bar{w}$ listed in Table III. From the analysis of the optimal weights, the first ten observations are immediately pinpointed as the ouliers in the data. The residual plot of the OWOLS estimator is shown in Fig. 3.

As a final example, we analyze a real data set of $N=23$ five-dimensional observations relating the aspect ratio, lift-to-drag ratio, weight (in pounds), maximal thrust and cost (units of $\$ 100000$ ) of 23 single-engine aircraft built over the years 1947-1979. The data are taken from [13] and listed in Table IV. Again in this case, we look for the $(n-1)$-dimensional affine set that explains the data. The application of the OWOLS regression yields the optimal weights reported in Table IV, and the regression parameters $A^{T}=[0.3474,-0.9375,0.0001,0.0000,0.0221], b=1.4022$. In Figs. 4 and 5, the residual plots of the OLS regression and the OWOLS regression are compared. From the plot in Fig. 5, we may conclude that, according to the OWOLS procedure, the observations 14, 20, and 22 may be considered as outliers, and a better fit is obtained by giving to these observations an appropriately low weight, see Table IV.

\section{CONCLUSIONS}

In this paper, we discussed the problem of affine orthogonal regression on multidimensional data contaminated by outliers. The proposed solution is based on a two-step procedure. The first step uses the RANSAC paradigm to determine a feasible set of initial weights and an estimate of outlier contamination. In the second step, a gradient based minimization is used to determine the optimal weights and compute the regression parameters. When the minimization step converges to the global optimum, the corresponding regression estimate is guaranteed to have up to 0.5 residual breakpoint. The optimal weights provide a global measure of the influence of the observations on the final estimation residual. We remark however that the convergence to the global optimum cannot in general be guaranteed, and that the robustness of the estimator strongly relies on the step one RANSAC phase. Tests performed on various real data sets confirmed the efficiency and reliability of the proposed method for data sets of medium/moderate size.

\section{APPENDIX \\ PROOF OF PROPOSITION 1}

We here present a Proof of Proposition 1 based on Lagrange multipliers. Let us rewrite (2), with the unit norm constraints on the columns of $A$

$$
\begin{array}{rl}
\min _{A, b} & f(A, b, X, w) \\
& =\sum_{i=1}^{N} w_{i}^{2}\left\|A^{T} x_{i}-b\right\|^{2} \\
& =\left\|\left(A^{T} X-b \iota^{\prime}\right) W\right\|_{F}^{2} \\
\text { subject to: } \quad a_{i}^{T} a_{i}=1, \quad i=1, \cdots, m .
\end{array}
$$


Letting $\Lambda=\operatorname{diag}\left\{\lambda_{1}, \lambda_{2}, \cdots, \lambda_{m}\right\}$ be a diagonal matrix having the Lagrange multipliers on the diagonal, the Lagrangian function for problem (18), (19) may be easily written as

$$
\mathcal{L}=f(A, b, X, w)-\operatorname{Tr}\left[\left(A^{T} A-I\right) \Lambda\right] .
$$

Substituting for $f$, the lagrangian can be rewritten as

$$
\begin{aligned}
\mathcal{L}= & \operatorname{Tr}\left[\left(A^{T} X-b u u^{T}\right) W^{2}\left(X^{T} A-u b^{T}\right)-A^{T} A \Lambda+\Lambda\right] \\
= & \operatorname{Tr}\left(A^{T} X W^{2} X^{T} A-2 A^{T} X W^{2} u b^{T}+\nu b b^{T}\right. \\
& \left.-A^{T} A \Lambda+\Lambda\right) .
\end{aligned}
$$

The necessary conditions for optimality require that the gradient of the lagrangian be zero (see for instance [10]), therefore

$$
\begin{aligned}
& \nabla_{A} \mathcal{L}=2 A^{T} X W^{2} X^{T}-2 b u^{T} W^{2} X^{T}-2 \Lambda A^{T}=0 \\
& \nabla_{b} \mathcal{L}=-2 A^{T} X W^{2} u+2 \nu b=0 .
\end{aligned}
$$

From (22) follows

$$
b=\frac{1}{\nu} A^{T} X W^{2} u
$$

that substituted in (21) yields

$$
A^{T} X\left(W^{2}-\frac{1}{\nu} W^{2} u u^{T} W^{2}\right) X^{T}-\Lambda A^{T}=0
$$

Defining $H$ as in (5), one gets

$$
H A-A \Lambda=0
$$

which says that the columns of $A$ must be $m$ eigenvectors of the symmetric matrix $H$, associated with some eigenvalues $\lambda_{1}, \lambda_{2}, \cdots, \lambda_{m}$, in order to satisfy the necessary conditions for optimality. Moreover, being $H$ symmetric, the eigenvectors of $H$ form an orthogonal set, and can always be scaled to have euclidean norm one, so to satisfy (19).

Evaluating now the objective (18) at the points that satisfy (23), (25), and (19), one gets

$$
\begin{aligned}
& f(A, b, X, w) \\
&=\operatorname{Tr}\left(A^{T} X W^{2} X^{T} A-\frac{1}{\nu} A^{T} X W^{2} u u^{T} W^{2} X^{T} A\right) \\
&=\operatorname{Tr}\left[A^{T} X\left(W^{2}-\frac{1}{\nu} W^{2} u u^{T} W^{2}\right) X^{T} A\right] \\
&=\operatorname{Tr}\left(A^{T} H A\right)=(\text { by }(25)) \\
& \operatorname{Tr}\left(A^{T} A \Lambda\right)=\left(\text { as } a_{i}^{T} a_{i}=1, \quad i=1, \cdots, m\right) \\
&= \sum_{i=1}^{m} \lambda_{i} .
\end{aligned}
$$

The objective function $f$ is then equal to the sum of the $m$ selected eigenvalues of $H$, which is clearly minimized if the first $m$ smallest eigenvalues of $H$ are selected. The optimal matrix
$A$ of the affine set must therefore contain by columns the $m$ orthonormal eigenvectors of $H$ associated to the first $m$ smallest eigenvalues of $H$. The $b$ parameter is then computed according to $(23)$.

\section{REFERENCES}

[1] D. L. Donoho and P. J. Huber, "The notion of breakdown point," in $A$ Festschrift for Erich Lehmann, P. Bickel, K. Doksum, and J. L. Hodges, Eds. Belmont, CA: Wadsworth, 1983.

[2] N. R. Draper and H. Smith, Applied Regression Analysis. New York: Wiley, 1966.

[3] M. A. Fischler and R. C. Bolles, "Random sample consensus: A paradigm for model fitting with applications to image anlaysis and automated cartography," Commun. ACM, vol. 24, no. 6, pp. 381-395, 1981.

[4] G.H. Golub and C. F. Van Loan, "An analysis of the total least squares problem," SIAM J. Numer. Anal., vol. 17, no. 6, pp. 883-893, 1980.

[5] F. R. Hampel, E. M. Ronchetti, and P. J. Rousseeuw, Robust Statistics: The Approach Based on Influence Functions. New York: Wiley, 1986.

[6] D. M. Hawkins, D. Bradu, and G. V. Kass, "Location of several outliers in multiple regression data using elemental sets," Technometrics, vol. 26, pp. 197-208, 1984.

[7] P.J. Huber, Robust Statistics. New York: Wiley, 1981.

[8] J. Juang, P. Ghaemmaghami, and K. B. Lim, "Eigenvalue and eigenvector derivatives of a nondefective matrix," J. Guidance, vol. 12, no. 4, pp. 480-486, 1989.

[9] B. Kamgar-Parsi, B. Kamgar-Parsi, and N. S. Netanyahu, "A nonparametric method for fitting a straight line to a noisy image," IEEE Trans. Pattern Anal. Mach. Intell., vol. 11, no. 9, pp. 998-1001, 1989.

[10] D. G. Luenberger, Linear and Nonlinear Programming. Reading, MA: Addison-Wesley, 1984, ch. 10.

[11] R. A. Maronna, O. Bustos, and V. Yohai, "Bias and efficiency robustness of general M-estimators for regression with random carriers," in Smoothing Techniques for Curve Estimation, T. Gasser and M. Rosenblatt, Eds. New York: Springer, 1993.

[12] P. Meer, D. Mintz, A. Rosenfeld, and D. Yoon Kim, "Robust regression methods for computer vision: a review," Int. J. Comput. Vis., vol. 6, no. 1, pp. 59-70, 1991.

[13] P. J. Rousseeuw and A. M. Leroy, Robust Regression and Outlier Detection. New York: Wiley, 1987.

[14] L. S. Shapiro and J. M. Brady, "Rejecting outliers and estimating errors in an orthogonal regression framework," Philos. Trans. R. Soc. London A, Math, Phys., Sci., vol. 350, pp. 407-439, 1995.

[15] P. H. S. Torr and D. W. Murray, "The development and comparison of robust methods for estimating the fundamental matrix," Int. J. Comput. Vis., vol. 24, no. 3, pp. 271-300, 1997.

[16] S. Ullman and R. Basri, "Recognition by linear combinations of models," IEEE Trans. Pattern. Anal. Mach. Intell., vol. 13, no. 10, pp. 992-1006, 1991.

[17] S. Van Huffel and J. Vandewalle, The Total Least Squares Problem: Computational Aspects and Analysis. Philadelphia, PA: SIAM, 1991.

[18] I. Weiss, "Line fitting in a noisy image," IEEE Trans. Pattern Anal. Mach. Intell., vol. 11, no. 3, pp. 325-329, 1989.

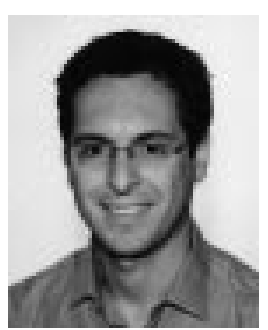

Giuseppe Carlo Calafiore was born in Torino, Italy, in 1969. He received the "Laurea" degree in electrical engineering in 1993, and the Ph.D. degree in information and system theory in 1997, both from Politecnico di Torino.

Since 1998, he has been Assistant Professor at Dipartimento di Automatica e Informatica, Politecnico di Torino. He held visiting positions at Information Systems Laboratory, Stanford University, Stanford, CA, in 1995, at Ecole Nationale Supérieure de Techniques Avenceés (ENSTA), Paris, in 1998, and at the University of California at Berkeley in 1999. His research interests are in the field of analysis, identification, and control of uncertain systems, pattern analysis and robotics, convex optimization, and randomized algorithms. 